\title{
Hofstadter-Hubbard model with opposite magnetic fields: Bardeen-Cooper-Schrieffer pairing and superfluidity in the nearly flat butterfly bands
}

\author{
M. Iskin \\ Department of Physics, Koç University, Rumelifeneri Yolu, 34450 Sarlyer, Istanbul, Turkey
}

(Received 11 September 2017; published 27 October 2017)

\begin{abstract}
Despite the multiband spectrum of the widely known Hofstadter butterfly, it turns out that the pairing correlations of the time-reversal-symmetric Hofstadter-Hubbard model are well described by a single order parameter that is uniform in real space. By exploiting a BCS mean-field theory for the nearly flat butterfly-bands regime of low magnetic-flux limit, here we reveal a number of unusual superfluid properties both in the ground state and at finite temperatures. Our thorough analysis includes but is not limited to the order parameter, condensate, and superfluid fractions, and the critical BCS and Berezinskii-Kosterlitz-Thouless transition temperatures.
\end{abstract}

DOI: 10.1103/PhysRevA.96.043628

\section{INTRODUCTION}

The Hofstadter model [1] consists only of a tight-binding kinetic term on a square lattice, where a spinless quantum particle is allowed to tunnel through nearest-neighbor sites with a hopping amplitude $t>0$, and meanwhile it gains an Aharonov-Bohm phase as a reflection of the magnetic vector potential. That is, the perpendicular magnetic field is taken into account via the well-established minimal coupling such that the particle acquires $e^{i 2 \pi \alpha}$ after traversing a loop around the unit cell. When the magnetic flux $\alpha$ corresponds to a ratio $p / q$ of relatively prime numbers $p$ and $q$, the single-particle energy spectrum $\varepsilon_{\mathbf{k} n}$ consists of $q$ bands that are indexed here by $n=0,1, \ldots, q-1$ in the first magnetic Brillouin zone. These subbands split from the tight-binding band of the flux-free case, and the energy versus $0 \leqslant \alpha \leqslant 1$ diagram reveals a self-similar fractal structure that is often called the Hofstadter butterfly. Since its original proposal back in 1976 [1], even though this simple model has continuously sustained the attention of a broad range of physicists and despite all the previous efforts, its intricate butterfly spectrum remains to be observed and utilized in a clear-cut fashion [2-4].

One of the historical drawbacks has been the competing length scales. This is because $\alpha$ is a direct measure of the ratio of the lattice spacing and the cyclotron radius of the charge carrier, leading to $\alpha \ll 1$ for typical electronic crystals even for the largest magnetic field ( $\sim 100$ Tesla) that is attainable in a laboratory. Thus, in order to access a sizable range of $\alpha$, one needs to have either an artificial lattice potential or an artificial magnetic field with high controllability. For instance, with the advent of artificial gauge fields in cold-atom experiments [5], $\alpha$ may be tuned at will in laser-generated optical lattices, which is one of the thriving themes in modern atomic and molecular physics. The current work is about the time-reversalsymmetric version of this model, which effectively describes a two-flavored quantum particle, e.g., a spin-1/2 fermion, with opposite magnetic fields for its flavors [6]. While such a setup sounds like a bizarre scenario for electronic systems, it has already been realized with cold atoms [7,8].

Motivated by this success [9-11], here we take advantage of the time-reversal symmetry that is manifested by the Hofstadter-Hubbard model with opposite magnetic fields, and develop a BCS mean-field theory for its low magnetic-flux regime. Given the simplicity of the spatially uniform superfluid
(SF) phase in this model, we believe it offers an ideal platform for studying the interplay of interactions and quantum Hall physics, e.g., the topological origin of the spin Hall insulator (SHI) lobes themselves, and the topological SHI-SF phase transitions are some of its notable outcomes. For instance, we show in this paper that the butterfly spectrum plays a major role in the weakly interacting regime such that the SF phase exhibits a number of unambiguous characteristic features both in the ground state and at finite temperatures. In the strongly interacting regime, however, this model effectively reduces to the usual Hubbard model without any additional physics. This is simply because once a Cooper pair is formed, its center-ofmass kinematics is neutral against the time-reversal-symmetric magnetic flux.

The rest of this paper is organized as follows. We first introduce the time-reversal-symmetric Hofstadter model in Sec. II A, and then derive a set of self-consistency equations in Sec. II B for the BCS mean field. The analysis of these equations is presented in Sec. III for the ground state, where we discuss the SF order parameter in Sec. III A, SHI-SF transition boundary in Sec. III B, condensate fraction in Sec. III C, and $\mathrm{SF}$ fraction in Sec. III D. Furthermore, a similar analysis is presented in Sec. IV for finite temperatures, where we discuss the critical BCS transition temperature in Sec. IV A and the critical Berezinskii-Kosterlitz-Thouless (BKT) transition temperature in Sec. IV B. The paper ends with a brief summary of our conclusions in Sec. V.

\section{MEAN-FIELD THEORY}

In this section, we first introduce the parameters of the model Hamiltonian and then derive a set of self-consistency equations that is based on the mean-field decoupling approximation for the BCS pairs.

\section{A. Hofstadter model with $\mathcal{T}$ symmetry}

It turns out that the Hofstadter butterfly spectrum is not only symmetric around zero energy due to the particle-hole symmetry of the parent Hamiltonian but it also has a mirror symmetry around $\alpha=0.5$. Thus, $\alpha=0.5$ corresponds to the maximally attainable magnetic flux within the model in such a way that the flux-free $\alpha=0$ system is identical to $\alpha=1$ [1]. In order to restore the time-reversal symmetry into 
the Hamiltonian, one considers a pseudo-spin-1/2 fermion experiencing an opposite magnetic flux for the components with an equal magnitude, i.e., $\alpha_{\uparrow}=-\alpha_{\downarrow}[6,9-11]$.

Given that the energy spectrum of the flux-free (to be more precise, either $q=\infty$ or $q=1)$ system $\varepsilon_{\mathbf{k}}=-2 t \cos \left(k_{x} a\right)-$ $2 t \cos \left(k_{y} a\right)$ is restricted to an energy window of $8 t$, where $\mathbf{k} \equiv$ $\left(k_{x}, k_{y}\right)$ is the crystal momentum and $a$ is the lattice spacing, and given the multiband structure of the butterfly for any finite $q$, the widths of the bands get progressively narrower with increasing $q$ from 2 . In turns out that the $n$th energy band is exponentially localized in energy near $\varepsilon_{n}$. This eventually leads to an infinite set of nearly flat butterfly bands in the $q \rightarrow \infty$ limit, recovering the quantum Hall regime of discrete Landau levels. Note the striking structural difference between the spectrum of the precise $q=\infty$ case and that of the $q \rightarrow \infty$ limit. Next we focus our previous analysis [11] of the timereversal-symmetric Hofstadter-Hubbard model to the large- $q$ regime, and explore its SF phase transition within the BCS mean-field pairing approximation.

\section{B. Self-consistency equations}

In the nearly flat butterfly-bands regime, where the bandwidths are assumed to be much smaller than the band gaps in such a way that $\varepsilon_{\mathbf{k} n} \rightarrow \varepsilon_{n}$ for every $\mathbf{k}$ state in all bands that are indexed here by $n=0,1, \ldots, q-1$ in the first magnetic Brillouin zone, the mean-field Hamiltonian $H$ per site can be written as

$$
\frac{H}{M}=\frac{1}{q} \sum_{n \sigma} \xi_{n} d_{n \sigma}^{\dagger} d_{n \sigma}-\frac{\Delta}{q} \sum_{n}\left(d_{n \uparrow}^{\dagger} d_{n \downarrow}^{\dagger}+\text { H.c. }\right)-\frac{\Delta^{2}}{U} .
$$

Here, $M$ is the number of lattice sites, $\xi_{n}=\varepsilon_{n}-\mu$ is the common flat-dispersion relation $\varepsilon_{n}$ shifted by the common chemical potential $\mu, d_{n \sigma}^{\dagger}\left(d_{n \sigma}\right)$ creates (annihilates) a $\sigma$ fermion in band $n$, and $\Delta=(|U| / q) \sum_{n}\left\langle d_{n \downarrow} d_{n \uparrow}\right\rangle$ is the order parameter characterizing a spatially uniform SF phase of $\mathcal{T}$-symmetric Cooper pairs that are made of $|n \uparrow,+\mathbf{k}\rangle$ and $|n \downarrow,-\mathbf{k}\rangle$ fermions with a stationary center of mass momentum. In addition, $U \leqslant 0$ is the on-site interaction between $\uparrow$ and $\downarrow$ fermions, $\langle\cdot\rangle$ denotes the thermal average, H.c. is the Hermitian conjugate, and $\Delta$ is assumed to be real without losing generality [11]. This is unlike the usual Hofstadter-Hubbard model with a broken $\mathcal{T}$ symmetry, where various competing SF phases require nontrivial sets of $q \times q$ order parameters, e.g., a vortex-lattice solution [12].

Thanks to the quadratic dependence on the fermion operators, it is a straightforward task to solve for the thermal properties of Eq. (1) via the minimization of the resultant thermodynamic potential. For instance, a self-consistent solution for $\Delta$ and $\mu$ can be obtained by solving the order parameter and number equations [13],

$$
\begin{aligned}
& \frac{1}{|U|}=\sum_{n} \frac{1}{2 q E_{n}} \tanh \left(\frac{E_{n}}{2 T}\right), \\
& F=1-\sum_{n} \frac{\xi_{n}}{q E_{n}} \tanh \left(\frac{E_{n}}{2 T}\right),
\end{aligned}
$$

for a given set of parameters. Here, the total particle filling $0 \leqslant F=N / M \leqslant 2$ per site is determined by $F=$
$(1 / q) \sum_{n \sigma}\left\langle d_{n \sigma}^{\dagger} d_{n \sigma}\right\rangle, E_{n}=\sqrt{\xi_{n}^{2}+\Delta^{2}}$ is the energy spectrum for the quasiparticles in band $n, k_{B} \rightarrow 1$ is the Boltzmann constant, and $T$ is the temperature. Note that the aforementioned particle-hole symmetry of the Hamiltonian manifests in Eqs. (2) and (3) around $\mu=0$ or, equivalently, half filling $F=1$.

When the strong-coupling condition $\Delta \gg t$ or, equivalently, $|U| \gg t$ is satisfied for all of the butterfly bands, we recover the familiar expressions of a flux-free system, e.g., $\Delta_{0}=\left(|U| / 2-4 t^{2} /|U|\right) \sqrt{F(2-F)}$ and $\mu=$ $-\left(|U| / 2-8 t^{2} /|U|\right)(1-F)$ are both proportional to the binding energy $(\propto|U|)$ of the Cooper pairs at $T=0$, leading eventually to $\Delta_{0}=(|U| / 2) \sqrt{F(2-F)}$ and $\mu=-(|U| / 2)(1-F)$ in the strict limit of structureless molecules. This is not surprising because once a Cooper pair is formed, its center-ofmass kinematics is neutral against the time-reversal-symmetric magnetic flux [11]. Alternatively, the only way a tightly bound molecule can move from a site $i$ to $j$ in lattice models is via the virtual ionization of its constituents, and given the binding energy $|U|$ as the penalty cost for breaking a pair, this leads to an effective hopping parameter $t_{i j m}=2 t_{i j \uparrow} t_{i j \downarrow} /|U|$ for the molecules [14]. Therefore, the effective hopping amplitude and magnetic flux of the molecules can be written as $t_{m}=$ $2 t^{2} /|U|$ and $\alpha_{m}=\alpha_{\uparrow}+\alpha_{\downarrow}=0$, respectively. Furthermore, these Cooper pairs are intrinsically hardcore by their composite nature as dictated by the Pauli exclusion principle in the $|U| / t \rightarrow \infty$ limit, and each site is either empty or singly occupied by one of them. These two local states may well be represented as an $\mathrm{SU}(2)$ algebra of the effective spin, and it turns out that the BCS mean field corresponds precisely to the classical approximation for the effective spin model in the molecular limit.

On the other hand, when $\mu=\varepsilon_{\bar{n}}$ coincides with one of the nearly flat butterfly bands at $U=0$, the strong-coupling condition $\Delta \gg t_{\bar{n}}^{\text {eff }}$ may immediately be satisfied for that particular band with a nonzero $U$ including the $U \rightarrow 0$ limit. This is because since the effective width of all of the bands $t_{n}^{\text {eff }}$ decreases dramatically from $t$ with increasing $q$ in the $q \gg 1$ regime, the strong-coupling condition $|U| \gg t_{\bar{n}}^{\text {eff }}$ can always be achieved for the $\bar{n}$ band with increasing $q$ no matter what $U$ is. However, this is not the case for the rest of the bands as they are energetically separated from the $\bar{n}$ band by varying single-particle gaps leading to $\left|\xi_{n \neq \bar{n}}\right| \gg \Delta$ when $\Delta \rightarrow 0$.

Next we reveal a number of unusual properties of the model Hamiltonian given in Eq. (1) by solving Eqs. (2) and (3) first in the ground state and then at finite temperatures.

\section{ANALYSIS OF THE GROUND STATE}

After setting $T=0$ in this section, we first discuss the characteristic features of the SF ground state near the SHI-SF transition boundary by a thorough analysis of the order parameter, and then explore its peculiar effects on the condensate and SF fractions.

\section{A. Order parameter}

The self-consistency Eqs. (2) and (3) for the ground state $\Delta_{0}$ and $\mu$, i.e., $1 /|U|=\sum_{n} 1 /\left(2 q E_{n}\right)$ and $F=1-$ $\sum_{n} \xi_{n} /\left(q E_{n}\right)$, are analytically tractable in two generic 


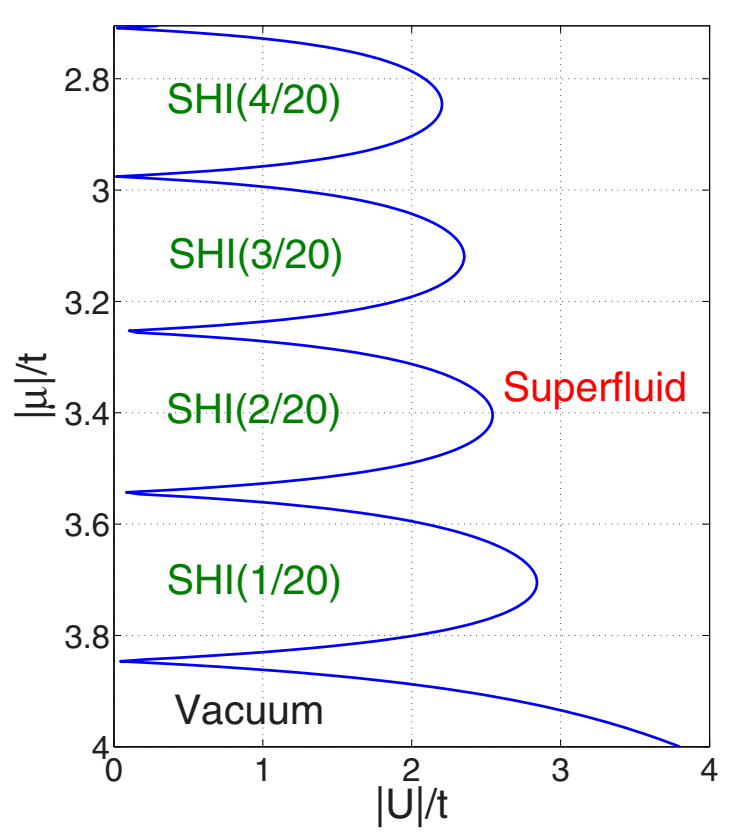

FIG. 1. The low-temperature $\left(T=10^{-4} t\right)$ phase diagram is shown for $\alpha=1 / 40$, whose SHI lobes closely resemble the wellknown MI lobes of a lattice Bose gas. Here, the total filling $F$ of each lobe is indicated in parentheses.

cases. For instance, when $\mu=\varepsilon_{\bar{n}}$ coincides with one of the nearly flat butterfly bands at $U=0$, we find $\Delta_{0}=$ $(|U| / 2) \sqrt{F_{\bar{n}}\left(2 / q-F_{\bar{n}}\right)}$ and $\mu_{0}=\varepsilon_{\bar{n}}+(|U| / 2)\left(F_{\bar{n}}-1 / q\right)$ for the $U \rightarrow 0$ limit [15]. We note that the total filling is given by $F=2 \bar{n} / q+F_{\bar{n}}$, where $0 \leqslant F_{\bar{n}}=1 / q-\xi_{\bar{n}} /\left(q E_{\bar{n}}\right) \leqslant 2 / q$ is the filling of the $\bar{n}$ th band, and that the particle-hole symmetry of the Hamiltonian manifests in the $\bar{n}$ th band around its half filling $F_{\bar{n}}=1 / q$ or, equivalently, $\mu=\varepsilon_{\bar{n}}$. Thus, unlike the usual BCS superfluids where $\Delta_{0}$ grows exponentially with $|U|$, here the growth is linear and much faster. As discussed towards the end of Sec. II B, while such a linear growth is quite typical for BCS superfluids in the strong-coupling or molecular limit, here it arises immediately with $U \neq 0$ due to the significant enhancement of the single-particle density of states in a nearly flat band. In addition, we find $\Delta=\Delta_{0}\left(1-2 e^{-\Delta_{0} / T}\right)$ as $T \rightarrow 0$.

On the other hand, when $\varepsilon_{\bar{n}-1}<\mu<\varepsilon_{\bar{n}}$ resides within one of the butterfly-band gaps at $U=0$, we find $\Delta_{0}=$ $\sqrt{\left(1 / U-1 / U_{c}\right) / C_{0}}$ and $F=2 \bar{n} / q$ for the $U \rightarrow U_{c}$ limit, where $1 / U_{c}=-\sum_{n} 1 /\left(2 q\left|\xi_{n}\right|\right)$ and $C_{0}=\sum_{n} 1 /\left(4 q\left|\xi_{n}\right|^{3}\right)$. Thus, there exists a critical interaction threshold $U_{c}$ for the SF phase transition when there is an energy gap for the single-particle excitations. This is illustrated in Fig. 1 for $\alpha=1 / 40$. Since the butterfly spectrum exhibits a self-similar structure with varying $\alpha$, the dependence of $U_{c}$ on $\mu, p$, or $q$ is an intricate one [11].

In particular, our main focus here is the SHI-SF transition boundary (e.g., see Fig. 1), which has the familiar shape of insulator lobes in the $\mu$ versus $|U|$ plane [11]. Note in Fig. 1 that $U_{c}$ is small but finite, even for $\mu=\varepsilon_{n}$ values, due to thermal effects. In order to gain some physical insight, next we obtain an approximate analytical expression for the tip of these SHI lobes when $q \gg 1$.

\section{B. SHI-SF transition}

First of all, the expression $1 / U_{c}=-\sum_{n} 1 /\left(2 q\left|\xi_{n}\right|\right)$ directly suggests that $U_{c} \rightarrow 0$ when $\mu=\varepsilon_{\bar{n}}$ coincides with one of the nearly flat butterfly bands at $U=0$, and that $U_{c} \approx-2 q\left|\xi_{\bar{n}}\right|$ when $\mu \rightarrow \varepsilon_{\bar{n}}$ from both below or above. In other words, the two consecutive SHI lobes, say $\bar{n}$ th lobe with filling $2(\bar{n}-1) / q$ and $(\bar{n}+1)$ th lobe with filling $2 \bar{n} / q$, touch each other precisely at $\mu=\varepsilon_{\bar{n}}$ when $U_{c}=0$. This leads to a symmetric and linear transition boundary in the $\mu$ versus $|U|$ plane, where the hole branch of the $\bar{n}$ th lobe and the particle branch of the $(\bar{n}+1)$ th lobe have exactly opposite slopes, $d \mu / d\left|U_{c}\right|= \pm 1 /(2 q)$, depending only on $q$. When $\alpha=1 / q$, since increasing $q$ rapidly flattens the lowest butterfly bands, the analysis given above is quite generic and applies even to small $q \gtrsim 8$ values [11]. When $\mu=0$ and $q$ is even, the physics is quite different [17].

Furthermore, it is possible to make analytical progress for the tip of the SHI lobes in the quantum Hall regime of discrete Landau levels based on the following analogy. Plugging the maximal density $\rho_{\max }=m \omega_{B} /(\hbar \pi)$ per Landau level into the maximal filling $F_{\max }=2 / q=\rho_{\max } a^{2}$ per butterfly band, and using $m=\hbar^{2} /\left(2 t a^{2}\right)$ for the effective mass of the particles in the $q \rightarrow \infty$ limit, we identify the band gap between the Landau levels as $A=\hbar \omega_{B}=4 \pi t / q$. Here, $\omega_{B}=q_{0} B / m$ is the cyclotron frequency of a charged particle with mass $m$ and charge $q_{0}$ in an external $\mathbf{B}=B \widehat{\mathbf{z}}$ field. While this expression is in perfect agreement with the first-order (in the small parameter $1 / q$ ) perturbative approach for the lowest butterfly-band gap when $\alpha=1 / q$ [18], the higher band gaps gradually deviate from this value for any finite $q$. In fact, it otherwise would give a uniform gap of the order of $8 t / q$, given that the total bandwidth $8 t$ is split into $q$ nearly flat butterfly bands. See Fig. 1, where $q=40$.

For the $q \gg 1$ regime, noting that the $n$th energy band is exponentially localized in energy near $\varepsilon_{n}$, here we assume $\varepsilon_{n} \approx-4 t+A(n+1 / 2)$, where the nearly flat butterfly bands are again indexed by $n=0, \ldots, q-1$. We note that since the exact bandwidths are not given by $A$ for finite $q$, this assumption clearly overestimates $\left|U_{c}\right|$ for all of the SHI lobes and, more importantly, it rapidly fails for the higher SHI lobes with increasing $\bar{n}$. Under this approximation, by plugging $\mu=-4 t+A s$ into the expression for $U_{c}$ to evaluate the tip of the $s=1,2, \ldots$ th SHI lobe, and using $\sum_{j=1}^{J} 1 /(2 j-1)$ $\approx[\ln (4 J)+\gamma] / 2$ for large $J$ with $\gamma \approx 0.577$ the Euler's constant, we obtain

$$
\left|U_{c}^{(s)}\right|=\frac{2 q A}{\gamma+\ln (4 q-4 s)+\sum_{j=1}^{s} \frac{2}{2 j-1}},
$$

leading to $\left|U_{c}^{(1)}\right| \approx 8 \pi t /(3.9635+\ln q)$ for the first lobe. In Table I, we compare this simple expression with that of the fully numerical calculation that is based on the exact butterfly spectrum. Note that four of the lowest SHI lobes are illustrated in Fig. 1 for $q=40$. While it is uplifting to see that the relative accuracy of the analytical expression improves monotonously with increasing $q$, the logarithmic convergence makes this progress very slow. Given the need for days-long computation times even for $q \sim 1000$, Eq. (4) may still offer an enormously convenient way for a reasonable estimate of $U_{c}$ in the large- $q$ regime [19]. 
TABLE I. Comparison of the tip of the first SHI lobe shows that the relative convergence between the analytical approximation given in Eq. (4) and the numerical result that is based on the exact butterfly spectrum is logarithmic.

\begin{tabular}{lccc}
\hline \hline$q$ & $\left|U_{c}^{(1)}\right| / t$ & $\left|U_{c}\right| / t$ & Error (\%) \\
\hline 40 & 3.284 & 2.841 & 15.6 \\
100 & 2.933 & 2.614 & 12.0 \\
1000 & 2.312 & 2.126 & 8.60 \\
2000 & 2.173 & 2.009 & 8.15 \\
5000 & 2.014 & 1.873 & 7.50 \\
\hline \hline
\end{tabular}

\section{Condensate fraction}

The on-site pairing parameter $\Psi_{i}=\left\langle c_{i \uparrow} c_{i \downarrow}\right\rangle$, where $c_{i \sigma}$ annihilates a $\sigma$ fermion on site $i$, characterizes the number of Cooper pairs in the usual way and it is directly related to the onsite SF order parameter $\Delta_{i}$ via $\Delta_{i}=U \Psi_{i}$. Since $\Delta_{i}$ is uniform in space in this paper, this leads to $\Delta=(|U| / M) \sum_{n \mathbf{k}} \Psi_{\mathbf{k} n}$ with the pair wave function $\Psi_{\mathbf{k} n}=\left[\Delta /\left(2 E_{\mathbf{k} n}\right)\right] \tanh \left[E_{\mathbf{k} n} /(2 T)\right]$ for a given $\mathbf{k}$ state in the first magnetic Brillouin zone. Thus, we identify $N_{c}=2 \sum_{n \mathbf{k}}\left|\Psi_{\mathbf{k} n}\right|^{2}$ as the total number of condensed particles in general [14], leading to $F_{c}=N_{c} / M=$ $(2 / q) \sum_{n}\left|\Psi_{n}\right|^{2}$ as their filling in the nearly flat butterfly-bands regime, where $\Psi_{\mathbf{k} n} \rightarrow \Psi_{n}$ for every $\mathbf{k}$ state of all butterfly bands.

The condensate filling $F_{c}=\left[\Delta_{0}^{2} /(2 q)\right] \sum_{n} 1 /\left(\xi_{n}^{2}+\Delta_{0}^{2}\right)$ for the ground state is also analytically tractable in two generic cases. When $\mu=\varepsilon_{\bar{n}}$ coincides with one of the nearly flat butterfly bands at $U=0$, we find $F_{c} \approx F_{\bar{n} c}=F_{\bar{n}}\left(2-q F_{\bar{n}}\right) / 2$ for the $U \rightarrow 0$ limit as long as $\Delta_{0} \neq 0$. Recall that the total particle filling is $F=2 \bar{n} / q+F_{\bar{n}}$ for this limit. Thus, $F_{\bar{n} c}$ is independent of $U$, and while all of the particles or holes in band $\bar{n}$ are condensed at its low- and full-filling regimes, where $F_{\bar{n} c} \rightarrow F_{\bar{n}}$ and $F_{\bar{n} c} \rightarrow 2 / q-F_{\bar{n}}$, respectively, only a half of the particles is condensed at half filling where $F_{\bar{n} c}=1 /(2 q)$. This is simply because, since the particles are strongly interacting in the $\bar{n}$ band as long as $\Delta_{0} \neq 0$ no matter how small it is, the fraction of condensed pairs in band $\bar{n}$ achieves its maximal value immediately with $U \neq 0$. This, however, is not the case for the rest of the particles in the lower bands as $\Delta_{0} \ll\left|\xi_{n \neq \bar{n}}\right|$ when $U \rightarrow 0$.

On the other hand, when $\varepsilon_{\bar{n}-1}<\mu<\varepsilon_{\bar{n}}$ resides within one of the butterfly-band gaps at $U=0$, we find $F_{c}=$ $\left(C_{1} / C_{0}\right)\left(1 / U-1 / U_{c}\right)$ for the $U \rightarrow U_{c}$ limit where $\Delta_{0} \ll\left|\xi_{n}\right|$ [20]. Here, $C_{0}=\sum_{n} 1 /\left(4 q\left|\xi_{n}\right|^{3}\right)$ and $C_{1}=\sum_{n} 1 /\left(2 q\left|\xi_{n}\right|^{2}\right)$. Recall that the total particle filling is $F=2 \bar{n} / q$ for this limit.

Thus, unlike the usual BCS superfluids where $F_{c} \propto \Delta_{0} / t$ grows exponentially slow with $|U|$ [14], here $F_{c}$ either immediately saturates or grows linearly with $|U|$, depending on where $\mu$ lies at $U=0$. This peculiar $U$ dependence is a direct reflection of that of $\Delta_{0}$ discussed in Sec. III A, and its effects are naturally not limited to the condensate fraction. For instance, next we show that $\Delta_{0}$ leaves its definitive signatures on the SF fraction as well.

\section{Superfluid fraction}

By definition, the SF density is directly proportional to the familiar phase stiffness of the effective phase-only
Hamiltonian, e.g., see Sec. IVB for a brief discussion about the critical BKT transition temperature. For instance, when $\mu=\varepsilon_{\bar{n}}$ coincides with one of the nearly flat butterfly bands at $U=0$, the phase stiffness is given by $\Gamma=(2 \bar{n}+$ 1) $q \Delta^{2} /(2 \pi|U|)$ for the $U \rightarrow 0$ limit [10], and it reduces to $\Gamma_{0}=(2 \bar{n}+1) F_{\bar{n}}\left(2-q F_{\bar{n}}\right)|U| /(8 \pi)$ at $T=0$. Substituting $t=\hbar^{2} /\left(2 m a^{2}\right)$ for the effective mass of the particles in the $q \rightarrow \infty$ limit and $\rho_{s}=F_{s} / a^{2}$ for the SF density into the relation $\Gamma=\hbar^{2} \rho_{s} /(4 m)$ shown in Sec. IV B, we obtain the SF fraction as $F_{s} / F=(2 \bar{n}+1) q F_{\bar{n}}\left(2-q F_{\bar{n}}\right)|U| /\left[4 \pi t\left(2 \bar{n}+q F_{\bar{n}}\right)\right]$. While this fraction becomes $F_{s} / F=\left(2-q F_{0}\right)|U| /(4 \pi t)$ for the $\bar{n}=0$ band with any given filling $0 \leqslant F_{0} \leqslant 2 / q$, it becomes $F_{S} / F=|U| /(4 \pi t)$ for any $\bar{n}$ band near their half fillings $F_{\bar{n}}=1 / q$. When $q$ is even, the latter $q$-independent expression may also be reproduced for the central bands by taking the $\mu=0$ or, equivalently, $F=1$ limit in the large- $q$ regime. Since the low-energy dispersion involves $q$ Dirac cones, it can be shown that $\Gamma_{0}=q \sqrt{\Delta_{0}^{2}+\mu^{2}} /(8 \pi)+$ $q \Delta_{0}^{2} \ln \left[\left(|\mu|+\sqrt{\Delta_{0}^{2}+\mu^{2}}\right) / \Delta_{0}\right] /(8 \pi|\mu|)$, for any $q$ in the weak-coupling limit [21]. This reduces to $\Gamma_{0}=q \Delta_{0} /(4 \pi)$ as $\mu \rightarrow 0$, leading eventually to $F_{s}=|U| /(4 \pi t)$ for the flattened centrally symmetric bands where $\Delta_{0}=|U| /(2 q)$ at their combined half filling.

Thus, unlike the usual BCS superfluids where the ground state of an entire Fermi gas turns from normal to SF immediately with $U \neq 0$ at $T=0$, i.e., $F_{s}=F$ in the $U \rightarrow 0$ limit, here the SF density sets in gradually with a linear growth in $|U|$. Having characterized the SF ground state near the SHI-SF transition boundary, next we discuss the SF phase at finite temperatures.

\section{CRITICAL TEMPERATURES}

After setting $T \neq 0$ and $\Delta \rightarrow 0$ in this section, we first discuss the characteristic features of the SF phase near the SF-normal transition boundary by a thorough analysis of the critical BCS temperature, and then compare it with the critical BKT temperature.

\section{A. BCS transition temperature}

The self-consistency Eqs. (2) and (3) for the critical BCS transition temperature $T_{c}$ and $\mu$, i.e., $1 /|U|=$ $\sum_{n} \tanh \left[\xi_{n} /\left(2 T_{c}\right)\right] /\left(2 q \xi_{n}\right)$ and $F=1-\sum_{n} \tanh \left[\xi_{n} /\left(2 q T_{c}\right)\right]$, are again analytically tractable in two generic cases. For instance, when $\mu=\varepsilon_{\bar{n}}$ coincides with one of the nearly flat butterfly bands at $U=0$, while we find $T_{c}=|U| /(4 q)$ and $\mu=\varepsilon_{\bar{n}}+2 T\left(q F_{\bar{n}}-1\right)$ near its half filling $F_{\bar{n}}=1 / q$ for the $U \rightarrow 0$ limit, we find $T_{c}=-|U| /\left[2 q \ln \left(q F_{\bar{n}} / 2\right)\right]$ in its lowfilling $q F_{\bar{n}} \rightarrow 0$ regime and $T_{c}=-|U| /\left[2 q \ln \left(1-q F_{\bar{n}} / 2\right)\right]$ in its full-filling $q F_{\bar{n}} \rightarrow 2$ regime. Note that while $T_{c} / \Delta_{0}=0.5$ is close to the well-known $\mathrm{BCS}$ ratio $T_{c} / \Delta_{0}=e^{\gamma} / \pi \approx 0.567$ near half filling, it diverges as $\lim _{x \rightarrow 0^{+}}-1 /(\sqrt{x} \ln x)$ in both of the low- and full-filling regimes. The latter is caused by the depletion of the low-energy density of states near the band edges, having more dramatic effects on the groundstate properties than the thermal ones. In addition, we find $\Delta=\sqrt{12} T_{c} \sqrt{1-T / T_{c}}$ for the $T \rightarrow T_{c}$ limit near half filling, leading to $\Delta=\sqrt{3} \Delta_{0} \sqrt{1-T / T_{c}}$. It is not only the precise form of this latter expression that is identical to that of the usual 
BCS one, but the prefactor $\sqrt{3} \approx 1.732$ also coincides with $e^{\gamma} \sqrt{8 /[7 \xi(3)]} \approx 1.736$, where $\xi(3) \approx 1.202$ is the Riemann- $\zeta$ function. While we are delighted by this almost perfect coincidence, it is probably an accidental match without any deep physical reasoning.

On the other hand, when $\varepsilon_{\bar{n}-1}<\mu<\varepsilon_{\bar{n}} \quad$ resides within one of the butterfly-band gaps at $U=0$, we find $\Delta=\left[\sum_{n} e^{-\left|\xi_{n}\right| / T_{c}} /\left(q C_{0} T_{c}\right)\right]^{1 / 2} \sqrt{1-T / T_{c}}$ for the $T \rightarrow T_{c}$ limit, leading to $\Delta=$ $\sqrt{U_{c}^{2} F /\left[2 T_{c}\left(U_{c}-U\right)\right]} \Delta_{0} \sqrt{1-T / T_{c}}$ in the $F \rightarrow 0$ regime, and $\Delta=\sqrt{U_{c}^{2}(2-F) /\left[2 T_{c}\left(U_{c}-U\right)\right]} \Delta_{0} \sqrt{1-T / T_{c}}$ in the $F \rightarrow 2$ regime. Thus, similar to the usual BCS superfluids near $T_{c}$, here $\Delta$ again decays as $\sqrt{1-T / T_{c}}$, which is one of the characteristic signatures of a second-order phase transition, but with a larger coefficient as $U \rightarrow U_{c}$. The large prefactor is again caused by the depleted density of states within the band gap as $\Delta_{0}$ is expected to be more susceptible to such changes than $T_{c}$.

All of these finite- $T$ results are based on mean-field theory, the equivalent of which is known to capture, quite accurately, the low- $T$ properties of a three-dimensional (3D) continuum Fermi gas in the weak-coupling BCS and strong-coupling BEC limits. In fact, this success has been the main motivation for its extensive use for the entire range of interactions, even though its accuracy is also known to be much less in the so-called BCS-BEC crossover. However, since the model at hand is a 2D lattice one with a complicated multi-flat-band energy spectrum, next we discuss the critical BKT temperature as a means to attest the validity of our finite- $T$ results.

\section{B. BKT transition temperature}

The critical BKT transition temperature $T_{\mathrm{BKT}}$ for a $2 \mathrm{D}$ $X Y$ model is given by the universal relation $T_{\mathrm{BKT}}=\pi \Gamma / 2$, where $\Gamma$ is the phase stiffness that is defined via the effective phase-only Hamiltonian $H_{X Y}=(\Gamma / 2) \int d^{2} \mathbf{r}\left(\nabla \theta_{\mathbf{r}}\right)^{2}$, under the assumption that the SF order parameter $\Delta_{\mathbf{r}}=\Delta e^{i \theta_{\mathbf{r}}}$ has a spatially varying phase. Setting $\theta_{\mathbf{r}}=\mathbf{K} \cdot \mathbf{r}$ for a uniform condensate density with $\hbar \mathbf{K}$ the pair momentum, we note that $H_{X Y} / A=\rho_{s} \hbar^{2} K^{2} /(4 m)=\rho_{s m} v^{2} / 2$, where $A$ is the area, $\mathbf{v}=\hbar \mathbf{K} /(2 m)$ is the velocity of the pairs, and $\rho_{s m}=m \rho_{s}$ is the SF-mass density. Thus, a self-consistent solution for $\Delta, \mu$, and $T_{\mathrm{BKT}}$ can be obtained by solving the order parameter and number equations given in Eqs. (2) and (3) together with the universal relation.

While the calculation of $\Gamma$ is typically a highly nontrivial task for an arbitrary $q$ value, it has recently been performed for the large- $q$ regime of our time-reversal-symmetric model [10]. For instance, when $\mu=\varepsilon_{\bar{n}}$ coincides with one of the nearly flat butterfly bands at $U=0$, such that $\left\{\left|\xi_{\bar{n}}\right|, T_{\mathrm{BKT}}\right\} \ll\left|\xi_{n \neq \bar{n}}\right|$, one finds

$$
\begin{gathered}
\frac{1}{|U|}=\frac{1}{2 q E_{\bar{n}}} \tanh \left(\frac{E_{\bar{n}}}{2 T_{\mathrm{BKT}}}\right), \\
F_{\bar{n}}=\frac{1}{q}-\frac{\xi_{\bar{n}}}{q E_{\bar{n}}} \tanh \left(\frac{E_{\bar{n}}}{2 T_{\mathrm{BKT}}}\right), \\
T_{\mathrm{BKT}}=\frac{(2 \bar{n}+1) q}{4|U|} \Delta^{2},
\end{gathered}
$$

for the $U \rightarrow 0$ limit. Here, the total particle filling is given by $F=2 \bar{n} / q+F_{\bar{n}}$, and this set of equations naturally gives $T_{\mathrm{BKT}} \leqslant T_{c}$ given that $T_{c}$ is determined by the $\Delta \rightarrow 0$ condition.

We note that the physical origins of $T_{c}$ and $T_{\mathrm{BKT}}$ are quite different. While $T_{c}$ has to do with the BCS pairing of particles or where $\mu$ lies within any of the given nearly flat butterfly bands, $T_{\mathrm{BKT}}$ has to do with the phase coherence and the true SF phase transition involving the entire Fermi gas. In other words, $T_{c}$ and $T_{\mathrm{BKT}}$, respectively, set the scale for the onset of Cooper pairing and the binding of vortex-antivortex pairs. This is the physical insight for the explicit $\bar{n}$ dependence appearing in Eq. (7). Next we show that while $T_{\mathrm{BKT}} \rightarrow T_{c}$ for $\bar{n} \gtrsim 4$ and $T_{\mathrm{BKT}} \lesssim T_{c}$ for $\bar{n} \lesssim 3$ near their half fillings $F_{\bar{n}}=1 / q$, we find $T_{\mathrm{BKT}} \ll T_{c}$ for any $\bar{n}$ if its filling $F_{\bar{n}}$ is sufficiently away from $1 / q$.

For instance, assuming $T_{\mathrm{BKT}} \approx T_{c}$, we may substitute the analytic $\Delta$ expression that is derived in Sec. IV A for the $T \approx T_{c}$ limit. This leads to $T_{\mathrm{BKT}} / T_{c}=(6 q \bar{n}+3 q) T_{c} /[(6 q \bar{n}+$ $\left.3 q) T_{c}+|U|\right]$, showing that the ratio approaches to unity if $T_{c} \gg|U| /(6 q \bar{n}+3 q)$. This last condition can be easily satisfied for large enough $\bar{n}$ around its half filling. To illustrate this, we plug the half-filling $T_{c}$ derived in Sec. IV A and obtain $T_{\mathrm{BKT}} / T_{c}=(6 \bar{n}+3) /(6 \bar{n}+7)$ for any $\bar{n}$. In comparison with the numerical findings 0.75 and 0.82 for the half-filled $\bar{n}=2$ and 3 bands [10], our analytical expression gives 0.79 and 0.84 . This good agreement suggests that our expression is quite accurate for $\bar{n} \gtrsim 4$ at their half fillings where $T_{\mathrm{BKT}}=$ $(6 \bar{n}+3)|U| /(24 q \bar{n}+28 q)$, and that $\bar{n}$ has to be very large for $T_{\mathrm{BKT}} \approx T_{c}$ away from their half fillings.

Similarly, assuming $T_{\mathrm{BKT}} \ll T_{c}$, we may substitute the analytic $\Delta_{0}$ expression that is derived in Sec. III A for the $T \ll$ $T_{c}$ limit. This leads to $T_{\mathrm{BKT}}=(2 \bar{n}+1) F_{\bar{n}}\left(2-q F_{\bar{n}}\right)|U| / 16$, which is independent of large $q$ at $\mu=0$ giving $T_{\mathrm{BKT}} \approx$ $|U| / 16$. Thus, we find $T_{\mathrm{BKT}} / T_{c}=(2 \bar{n}+1) / 4$ for half filling, which coincides exactly with the numerical finding $1 / 4$ for the $\bar{n}=0$ [10] band. This perfect agreement may further suggest that our expression $T_{\mathrm{BKT}}=F_{0}\left(2-q F_{0}\right)|U| / 16$ for the $\bar{n}=0$ band may work well for all other fillings as well. As $\bar{n}$ increases, $T_{\mathrm{BKT}}$ eventually approaches to $T_{c}$. For instance, away from half filling, the ratio $T_{\mathrm{BKT}} / T_{c}=$ $(2 \bar{n}+1) q F_{\bar{n}} \ln \left[2 /\left(q F_{\bar{n}}\right)\right]$ ultimately vanishes in the $q F_{\bar{n}} \rightarrow$ 0 limit for any finite $\bar{n}$. Thus, we conclude that while $T_{\mathrm{BKT}}=(2 \bar{n}+1) F_{\bar{n}}|U| / 8$ in the low-filling regime, $T_{\mathrm{BKT}}=$ $(2 \bar{n}+1)\left(2 / q-F_{\bar{n}}\right)|U| / 8$ in the full-filling one.

We note in passing that when the strong-coupling condition $|U| \gg t$ is satisfied for all of the butterfly bands, one must recover the familiar expressions of a flux-free system, where $T_{c} \propto|U|$ is proportional to the binding energy $|U|$ and $T_{\mathrm{BKT}} \propto$ $t^{2} /|U|$ is proportional to the effective mass $\hbar^{2} /\left(2 t_{m} a^{2}\right)$ of the structureless molecules, leading to $T_{\mathrm{BKT}} \ll T_{c}$ as well. Having characterized the SF phase at finite temperatures, we end this paper with a brief summary of our conclusions.

\section{CONCLUSIONS}

To summarize, we took advantage of the time-reversal symmetry that is manifested by the Hofstadter-Hubbard model with opposite magnetic fields, and developed a BCS mean-field theory for its multiband spectrum in the nearly flat butterfly-bands regime. In particular, our detailed analysis 
that includes but is not limited to the order parameter, condensate, and superfluid fractions, and the critical BCS and BKT transition temperatures revealed a number of unusual characteristics for the SF transition both in the ground state and at finite temperatures.

Given the simplicity of the spatially uniform SF phase in this time-reversal-symmetric model, e.g., without any serious complications coming from the competing phases such as vortexlike excitations, and its direct relevance to Lieb-like lattice models exhibiting flat bands, we believe the model offers an ideal platform for studying the interplay between the pairing correlations and the topologically nontrivial butterfly bands. For instance, a rich variety of topological phases and topological phase transitions have recently become some of the central topics in modern condensed-matter physics, and both the topological nature of the SHI lobes themselves and the topological SHI-SF phase transitions are a few of the standout results. The unambiguous signatures that are highlighted in this work not only guide the way in shaping our intuition behind these competing phases, but they also serve as ultimate benchmarks for numerically exact quantum Monte Carlo simulations.

\section{ACKNOWLEDGMENT}

The author acknowledges funding from TÜBITAK and the BAGEP award of the Turkish Science Academy, and the discussions with R. O. Umucalılar.
[1] D. R. Hofstadter, Energy levels and wave functions of Bloch electrons in rational and irrational magnetic fields, Phys. Rev. B 14, 2239 (1976).

[2] U. Kuhl and H.-J. Stöckmann, Microwave Realization of the Hofstadter Butterfly, Phys. Rev. Lett. 80, 3232 (1998).

[3] C. R. Dean, L. Wang, P. Maher, C. Forsythe, F. Ghahari, Y. Gao, J. Katoch, M. Ishigami, P. Moon, M. Koshino, T. Taniguchi, K. Watanabe, K. L. Shepard, J. Hone, and P. Kim, Hofstadter's butterfly and the fractal quantum Hall effect in moiré superlattices, Nature (London) 497, 598 (2013).

[4] L. A. Ponomarenko, R. V. Gorbachev, G. L. Yu, D. C. Elias, R. Jalil, A. A. Patel, A. Mishchenko, A. S. Mayorov, C. R. Woods, J. R. Wallbank, M. Mucha-Kruczynski, B. A. Piot, M. Potemski, I. V. Grigorieva, K. S. Novoselov, F. Guinea, V. I. Fal'ko, and A. K. Geim, Cloning of Dirac fermions in graphene superlattices, Nature (London) 497, 594 (2013).

[5] J. Dalibard, F. Gerbier, G. Juzeliŭnas, and P. Öhberg, Colloquium: Artificial gauge potentials for neutral atoms, Rev. Mod. Phys. 83, 1523 (2011).

[6] D. Cocks, P. P. Orth, S. Rachel, M. Buchhold, K. Le Hur, and W. Hofstetter, Time-Reversal-Invariant Hofstadter-Hubbard Model with Ultracold Fermions, Phys. Rev. Lett. 109, 205303 (2012).

[7] M. Aidelsburger, M. Atala, M. Lohse, J. T. Barreiro, B. Paredes, and I. Bloch, Realization of the Hofstadter Hamiltonian with Ultracold Atoms in Optical Lattices, Phys. Rev. Lett. 111, 185301 (2013).

[8] C. J. Kennedy, G. A. Siviloglou, H. Miyake, W. C. Burton, and W. Ketterle, Spin-Orbit Coupling and Spin Hall Effect for Neutral Atoms Without Spin-Flips, Phys. Rev. Lett. 111, 225301 (2013).

[9] L. Wang, H.-H. Hung, and M. Troyer, Topological phase transition in the Hofstadter-Hubbard model, Phys. Rev. B 90, 205111 (2014).

[10] S. Peotta and P. Törmä, Superfluidity in topologically nontrivial flat bands, Nat. Commun. 6, 8944 (2015); L. Liang, T. I. Vanhala, S. Peotta, T. Siro, A. Harju, and P. Törmä, Band geometry, Berry curvature, and superfluid weight, Phys. Rev. B 95, 024515 (2017).

[11] R. O. Umucalılar and M. Iskin, BCS Theory of Time-ReversalSymmetric Hofstadter-Hubbard Model, Phys. Rev. Lett. 119, 085301 (2017).
[12] R. O. Umucalılar and M. Iskin, Superfluid transition in the attractive Hofstadter-Hubbard model, Phys. Rev. A 94, 023611 (2016).

[13] These equations are simply reduced from the general expressions given in Ref. [11], where the extra factor of $q$ appearing in the denominator of Eq. (3) is a typographical error there.

[14] M. Iskin and C. A. R. Sá de Melo, Quantum phases of FermiFermi mixtures in optical lattices, Phys. Rev. A 78, 013607 (2008).

[15] In the quantum Hall regime of discrete Landau levels, our halffilling expression $\Delta_{0}=|U| /(2 q)$ precisely recovers the continuum model of Ref. [16], where they find $\Delta_{0}=m|g| \omega_{B} /(4 \pi \hbar)$ with $m=\hbar^{2} /\left(2 t a^{2}\right)$ and $\hbar \omega_{B}=4 \pi t / q$ for the $g=U a^{2} \rightarrow 0$ limit.

[16] T. Anzai and Y. Nishida, Two-dimensional Fermi gas in antiparallel magnetic fields, Phys. Rev. A 95, 051603 (2017).

[17] When $\mu=0$ and $q$ is even, the centrally symmetric energy bands $(n=q / 2$ and $q / 2-1)$ touch each other at zero energy, forming $q$ Dirac cones in the first magnetic Brillouin zone. We have shown previously that [11] the $\left|U_{c}\right|$ versus $\alpha$ phase diagram exhibits an oscillatory pattern, repeating itself in a self-similar structure, due to the fractal nature of the spectrum. The oscillations are between the $\alpha$ interval $\left(1 / 2^{+}, 1 / 4^{-}\right),\left(1 / 4^{+}, 1 / 6^{-}\right)$, etc. because as we approach $1 / q$ ratios with large $p$, there are more and more Dirac cones gradually turning the semimetal phase into a normal metal where $U_{c} \rightarrow 0$. It is such that for any given prime number $p$, each oscillation consists of $p-1$ data points, which can be written in the form $\alpha_{j j^{\prime}}=p /\left(2 p j+2 j^{\prime}\right)$ where $j=1,2, \ldots$ labels the oscillation between the $\alpha$ interval $1 /(2 j)^{+}$and $1 /(2 j+2)^{-}$, and $j^{\prime}=1,2, \ldots, p-1$. If $p$ is not a prime number, there are clearly fewer data points. For instance, $p=9$ has $8-2=6$ data points since $9 / 24=3 / 8$ and $9 / 30=3 / 10$ in the first oscillation.

[18] F. Harper, S. H. Simon, and R. Roy, Perturbative approach to flat Chern bands in the Hofstadter model, Phys. Rev. B 90, 075104 (2014).

[19] Equation (4) can be mapped to that of the continuum model of Ref. [16] after setting $U=g / a^{2}$ and $2 \pi \alpha=\Phi / \phi_{0}$, where $\alpha=1 / q, \Phi=B a^{2}$ is the flux per unit cell, and $\phi_{0}=\hbar / q_{0}$ is the flux quantum. This leads to $2 m\left|g_{c}^{(s)}\right|=8 \pi /\left[\gamma+\ln \left(8 \pi \phi_{0} / \Phi-\right.\right.$ $\left.4 s)+\sum_{j=1}^{s} 2 /(2 j-1)\right]$ for the tip of the $s$ th lobe located at $\mu=\hbar \omega_{B} s$. Furthermore, if the tip of the first SHI lobe $g_{c}^{(1)}$ is 
known, then the tips of the higher lobes can be generated by $g_{c}^{(s)} \approx 1 /\left\{1 / g_{c}^{(1)}+\sum_{j=2}^{s} m /[2 \pi(2 j-1)]\right\}$.

[20] In comparison, we find that the total condensate filling grows as $F_{c}=1 /(2 C)\left(1 / U-1 / U_{c}\right)^{2}$ in the $\Delta_{0} \rightarrow 0$ limit when $\mu=0$ and $q$ is even. Here, $C$ is the slope of the low-energy density of states near the Dirac cones, i.e., $C|\varepsilon|$ as $\varepsilon \rightarrow 0$, and $U_{c}$ is the critical threshold for the semimetal-SF transition [11].

[21] Setting $q=2$ for graphene, it reduces exactly to the expression given in N. B. Kopnin and E. B. Sonin, Supercurrent in superconducting graphene, Phys. Rev. B 82, 014516 (2010). 